\title{
Measuring dopant concentrations in compensated $p$-type crystalline silicon via iron-acceptor pairing
}

\author{
D. Macdonald, ${ }^{1, a)}$ A. Cuevas, ${ }^{1}$ and L. J. Geerligs ${ }^{2}$ \\ ${ }^{1}$ Department of Engineering, College of Engineering and Computer Science, Australian National University, \\ Canberra, Australian Capital Territory 0200, Australia \\ ${ }^{2}$ ECN Solar Energy, P.O. Box 1, NL-1755 ZG Petten, The Netherlands
}

(Received 10 April 2008; accepted 24 April 2008; published online 23 May 2008)

\begin{abstract}
We present a method for measuring the concentrations of ionized acceptors and donors in compensated $p$-type silicon at room temperature. Carrier lifetime measurements on silicon wafers that contain minute traces of iron allow the iron-acceptor pair formation rate to be determined, which in turn allows the acceptor concentration to be calculated. Coupled with an independent measurement of the resistivity and a mobility model that accounts for majority and minority impurity scatterings of charge carriers, it is then possible to also estimate the total concentration of ionized donors. The method is valid for combinations of different acceptor and donor species. (C) 2008 American Institute of Physics. [DOI: 10.1063/1.2936840]
\end{abstract}

Low-cost silicon feedstock will most likely become an important source of silicon for photovoltaics over the coming years. Due to the relative difficulty of removing dopant species during purification, this low-cost material is likely to be quite strongly compensated, meaning that it may contain significant quantities of both $n$ - and $p$-type dopants. This can in turn impact device performance, for example, through reduced carrier mobilities. The concentrations of dopants in compensated material can be determined via lowtemperature photoluminescence ${ }^{1,2}$ (PL) or infrared absorption (IR) measurements. ${ }^{3,4}$ Both methods require low temperatures (between 4.2 and $15 \mathrm{~K}$ ) and are mostly used for dopant concentrations below $10^{16} \mathrm{~cm}^{-3}$, for which they are well characterized but which is perhaps too low for some compensated solar-grade silicon. Higher dopant concentrations may also be measured by physical techniques such as secondary ion mass spectroscopy or glow-discharge mass spectroscopy. However, these techniques measure total concentrations, not only the electrically active component, and are not commonly available in photovoltaic laboratories (as is also true for low-temperature PL and IR).

Here, we present an alternative method for determining the concentration of electrically active acceptors in compensated $p$-type silicon based on carrier lifetime measurements of the formation rate of iron-acceptor pairs at room temperature. The concentration of electrically active donors can then also be inferred. This method for determining the acceptor concentration has been verified with measurements on noncompensated materials.

The technique is based on the well-known pairing behavior of iron-acceptor pairs in silicon. ${ }^{5-7}$ After the ironacceptor pairs are dissociated, usually by strong illumination, they reassociate with a time constant $\tau_{\text {assoc }}$ given by ${ }^{8}$

$$
\tau_{\text {assoc }}=B \frac{T}{N_{A}} \exp (0.66 \mathrm{eV} / k T),
$$

where the constant $B=5.0 \times 10^{5} \mathrm{~s} / \mathrm{K} \mathrm{cm}^{3}$, the temperature $T$ is in Kelvin ( $304 \mathrm{~K}$ was used in this study), $N_{A}$ is the acceptor concentration $\left(\mathrm{cm}^{-3}\right)$, Boltzmann's constant $k=8.617$

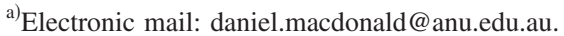

$\times 10^{-5} \mathrm{eV} / \mathrm{K}$, and $\tau_{\text {assoc }}$ is in units of seconds. The time constant $\tau_{\text {assoc }}$ can be accurately determined by measuring the change in carrier lifetime as iron-acceptor pairs re-form after dissociation, and as demonstrated below. It is then a simple matter to calculate $N_{A}$. The efficacy of this technique is due to the fact that in any $p$-type silicon wafer, even if heavily compensated, interstitial iron will exhibit a positive charge, and therefore readily form pairs with any negatively charged ionized acceptors. The repairing rate is determined by both the concentration of those acceptors and the migration enthalpy of the diffusing species, ${ }^{9}$ in this case interstitial iron. However, it is not affected by the presence of compensating dopants or by the reduction in the majority carrier concentration that they cause. However, in $n$-type silicon (compensated or not), the charge state of interstitial iron is neutral, iron-acceptor pairs will not form, ${ }^{5}$ and this method for determining $N_{A}$ cannot be applied.

The wafers used in this study were from five different $p$-type ingots grown by the Czochralski $(\mathrm{Cz})$ method. Three ingots were doped with boron only, while two were doped with both boron and phosphorus. The amounts of dopant added to the silicon melt for each ingot are shown in Table I, while the actual dopant concentrations incorporated in the wafers are affected by segregation and evaporation during ingot growth. They will vary from somewhat less than the initial concentration in the melt to somewhat more than the initial melt concentration depending on which part of the ingot the wafers are taken from, as discussed in more detail below. Nevertheless, the concentrations added to the melt provide an approximate guide to the dopant concentrations that can be expected in the wafers. High-purity feedstock and dopant sources were used during ingot growth; hence, we do not expect significant quantities of unintended dopants. The interstitial oxygen concentrations were measured by room temperature Fourier-transform IR spectroscopy ${ }^{10}$ and were between 18 and $23 \mathrm{ppm}$ by atom. Some samples from each ingot were annealed in nitrogen for $35 \mathrm{~min}$ at $700{ }^{\circ} \mathrm{C}$ to remove possible oxygen-related thermal donors, ${ }^{11,12}$ although these samples were subsequently found to exhibit no measurable differences to similar nonannealed samples. The samples contained trace quantities of interstitial iron, in the 
TABLE I. Parameters for the five ingots studied: dopant concentrations added to the melt, measured resistivities, and the corresponding boron concentrations $N_{A}$ for the noncompensated control ingots, measured iron-acceptor repairing time constants $\tau_{\text {assoc }}$, the corresponding boron concentrations $N_{A}$, the estimated donor concentrations $N_{D}$, and the hole mobilities determined by using the model of Reggiani et al.

\begin{tabular}{|c|c|c|c|c|c|c|c|c|}
\hline Ingot & $\begin{array}{c}\text { Boron } \\
\text { added to melt } \\
\left(10^{16} \mathrm{~cm}^{-3}\right)\end{array}$ & $\begin{array}{l}\text { Phosphorus } \\
\text { added to melt } \\
\left(10^{16} \mathrm{~cm}^{-3}\right)\end{array}$ & $\begin{array}{c}\text { Measured } \\
\text { resistivity } \\
\rho(\Omega \mathrm{cm})\end{array}$ & $\begin{array}{l}N_{A} \text { from } \\
\text { resistivity } \\
\left(10^{16} \mathrm{~cm}^{-3}\right)\end{array}$ & $\tau_{\text {assoc }}(\mathrm{s})$ & $\begin{array}{c}N_{A} \text { from } \\
\tau_{\text {assoc }}\left(10^{16} \mathrm{~cm}^{-3}\right)\end{array}$ & $\begin{array}{c}\text { Estimated } \\
N_{D}\left(10^{16} \mathrm{~cm}^{-3}\right)\end{array}$ & $\begin{array}{c}\text { Hole } \\
\text { mobility } \\
\mu_{h}\left(\mathrm{~cm}^{2} / \mathrm{V} \mathrm{s}\right)\end{array}$ \\
\hline 74 & 0.35 & Nil & 4.4 & 0.32 & 3700 & 0.36 & Nil & 440 \\
\hline 72 & 1.75 & Nil & 1.0 & 1.5 & 800 & 1.7 & Nil & 410 \\
\hline 73 & 5.0 & Nil & 0.40 & 4.3 & 310 & 4.3 & Nil & 367 \\
\hline 45 & 3.25 & 2.75 & 1.6 & $\cdots$ & 330 & 4.0 & 2.9 & 362 \\
\hline 44 & 7.5 & 5.0 & 0.47 & $\ldots$ & 165 & 8.1 & 4.0 & 323 \\
\hline
\end{tabular}

range $(2-6) \times 10^{10} \mathrm{~cm}^{-3}$, as detected by carrier lifetime measurements. ${ }^{6,13}$ Surface passivation for sensitive bulk lifetime measurement was achieved by plasma-enhanced chemical vapor deposited silicon nitride films on both surfaces after surface etching and cleaning steps.

Effective carrier lifetimes were measured by using the quasi-steady-state photoconductance (QSSPC) technique. ${ }^{14}$ Lifetimes were extracted at an excess carrier density of $\Delta n$ $=1 \times 10^{15} \mathrm{~cm}^{-3}$ in most cases. Iron-acceptor pairs were dissociated prior to lifetime measurements by illumination for $30 \mathrm{~s}$ with strong white light (approximately $100 \mathrm{~mW} / \mathrm{cm}^{2}$ ) from a halogen lamp. Note that it is not essential that all iron-acceptor pairs are dissociated since this will not affect the determination of the association time constant $\tau_{\text {assoc }}$. Resistivities were determined from dark conductance measurements performed with the QSSPC apparatus.

Figure 1 shows a typical lifetime decrease as the ironboron pairs re-form, in this case for a $1.6 \Omega \mathrm{cm}$ compensated sample. The solid line shows an exponential fit to the data and yields a time constant of $\tau_{\text {assoc }}=330 \mathrm{~s}$. Using Eq. (1), this corresponds to an acceptor concentration of $N_{A}=4.0$ $\times 10^{16} \mathrm{~cm}^{-3}$. Figure 2 shows similar results for several compensated and noncompensated samples, recast as relative interstitial iron concentrations $\left[\mathrm{Fe}_{i}\right]=1 / \tau-1 / \tau_{\text {final }}$, where $\tau_{\text {final }}$ is the lifetime after all of the pairs have re-formed. In this case, the exponential fits appear as straight lines, allowing simpler extraction of the time constants $\tau_{\text {assoc. }}$. We estimate the uncertainty in repeated lifetime measurements on a single sample to be less than $\pm 3 \%$. Fitting an exponential decay to lie within the uncertainty range of the lifetime data yields $\tau_{\text {assoc }}$ values that are typically uncertain by about $\pm 10 \%-15 \%$, unless there is so little iron as to make the lifetime changes almost undetectable. Additional uncertain-

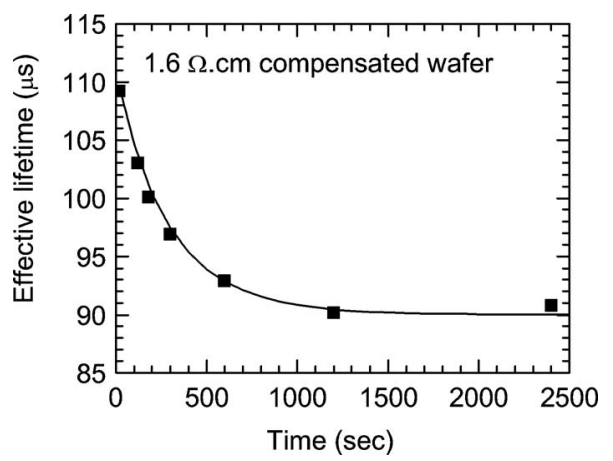

FIG. 1. Reduction in lifetime as a function of time for a $1.6 \Omega \mathrm{cm}$ compensated wafer as FeB pairs re-form after dissociation by illumination. The lifetime was measured at an excess carrier density of $1 \times 10^{15} \mathrm{~cm}^{-3}$. The

solid line shows an exponential fit with a time constant $\tau_{\text {assoc }}=330 \mathrm{~s}$. exponential fits, with the time constants $\tau_{\text {assoc }}$ also shown.
Downloaded 02 Mar 2009 to 150.203.161.6. Redistribution subject to AlP license or copyright; see http://apl.aip.org/apl/co ties in the temperature and other wafer parameters mean that the determined values of $N_{A}$ are uncertain by approximately $20 \%$ in most cases.

Table I lists the values of $\tau_{\text {assoc }}$ and the corresponding $N_{A}$ values for each of the ingots studied. Also shown for the noncompensated ingots are the $N_{A}$ values calculated from the measured resistivities $\rho$ via $\rho=q \mu_{h} p_{0}$, where $q$ is the electronic charge, $\mu_{h}$ the majority hole mobility, and $p_{0}$ the equilibrium hole concentration. For the noncompensated ingots, $p_{0}=N_{A}$. The mobilities were determined self-consistently by using the model of Reggiani et al. ${ }^{15}$ The more complex mobility model of Klaassen ${ }^{16,17}$ yields almost identical results for $N_{A}$. Note that for the three non-compensated control ingots, the $N_{A}$ values determined via iron-acceptor pairing are in good agreement with the values determined via the measured resistivities. This indicates that the iron-acceptor pairing approach is an accurate way to determine $N_{A}$. The values are also in good agreement with the concentrations of boron added to the melt, although these provide only an approximate guide due to the effects of segregation, as discussed below.

Taking the values for $N_{A}$ as determined by iron-acceptor pairing in the compensated ingots, we may then also estimate the ionized donor concentration $N_{D}$ in these samples. Assuming that $p_{0}=N_{A}-N_{D}$ and again by using the mobility model of Reggiani et al. ${ }^{15}$ to estimate the hole mobility in the presence of both $n$ - and $p$-type dopants, we can adjust $N_{D}$ and the corresponding $\mu_{h}$ until the resulting resistivity $\rho=q \mu_{h} p_{0}$ is in agreement with the measured value. Note that there are some relatively small differences between majority carrier mobilities determined with either the model of Reggiani et al. or Klaassen's model when both donor and acceptor dopants are

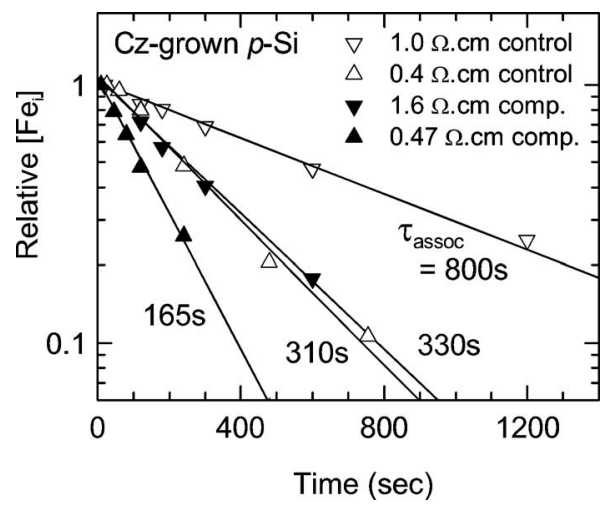

FIG. 2. Exponential decays of the relative interstitial iron concentration for several compensated and noncompensated samples. The solid lines show

exponential fits, with the time constants $\tau_{\text {assoc }}$ also shown. 
present. However, lack of experimental data for mobilities in compensated silicon makes it difficult to be sure which model is more accurate. In any case, the choice of the model makes little difference to the values of $N_{D}$ determined here. The values of $N_{D}$ determined in this way are shown in Table I. Again, they are in quite good agreement with the concentrations of dopant added to the melt. Note that the uncertainty in the estimated values of $N_{D}$ will be somewhat larger than the uncertainty in $N_{A}$ since it is compounded by uncertainties in the measured resistivity and the mobility model.

It is possible to check if the results are consistent with the amounts of dopants added to the melt and the impact of segregation during ingot growth. Assuming segregation coefficients of 0.75 for boron and 0.35 for phosphorus, and ignoring any evaporation of dopants from the melt, wafers cut from ingot number 45 would have $4.0 \times 10^{16} \mathrm{~cm}^{-3}$ of boron and $3.5 \times 10^{16} \mathrm{~cm}^{-3}$ of phosphorus if they were sliced from the region where $86 \%$ of the melt had already crystallized. For ingot number 44 , there would be $8.1 \times 10^{16} \mathrm{~cm}^{-3}$ of boron and $4.6 \times 10^{16} \mathrm{~cm}^{-3}$ of phosphorus if $77 \%$ of the melt had crystallized. These values are within the estimated uncertainty range of $\pm 20 \%$ of the measured values, indicating that the results are consistent with the expected segregation of dopants during ingot growth.

It is worth noting that, although boron was the acceptor species used in the samples here, the method should in principle work equally well for other acceptors, or indeed a mix of acceptors, since the repairing kinetics are independent of the acceptor species. ${ }^{8}$ The technique for determining $N_{A}$ via iron-acceptor pairing is also independent of the carrier mobilities (which are likely to be altered in compensated material) since it is only the characteristic decay time of the carrier lifetime as the pairs re-form that is required, and this is determined by the movement of interstitial iron, not the charge carriers. In other words, it is not necessary to know the absolute magnitude of the lifetime itself nor the absolute value of the carrier density at which it is extracted (although the latter must be kept constant), both of which are affected by the mobilities in the QSSPC technique. Only relative lifetime changes are required. On the other hand, estimating $N_{D}$ via the resistivity, as described above, does require knowledge of the hole mobility.
Finally, it may seem restrictive to require interstitial iron to be present in a sample for the technique to be applicable. However, we have found suitable traces of iron in almost all $\mathrm{Cz}$-grown $p$-type wafers we have measured and also in many float-zone wafers. Multicrystalline silicon wafers grown for solar cells always contain sufficient interstitial iron for these purposes, at least in the as-cut state, courtesy of contamination from the crucible. ${ }^{18}$ The relatively straightforward method presented here for measuring $N_{A}$ and then estimating $N_{D}$ in compensated $p$-type silicon is likely to be useful in future studies of relatively "dirty" silicon for solar cells.

D.M. is supported by an Australian Research Council fellowship. L.J.G. would like to acknowledge SenterNovem for support. The authors are grateful to K. Petter of Q-Cells for providing the silicon wafers and to Eugenia Sepulveda for assistance with sample preparation.

${ }^{1}$ P. M. Colley and E. C. Lightowlers, Semicond. Sci. Technol. 2, 157 (1987).

${ }^{2}$ G. Davies, Phys. Rep. 176, 83 (1989).

${ }^{3}$ B. O. Kolbesen, Appl. Phys. Lett. 27, 353 (1975).

${ }^{4}$ S. C. Baber, Thin Solid Films 72, 201 (1980).

${ }^{5}$ L. C. Kimerling and J. L. Benton, Physica B \& C 116, 297 (1983).

${ }^{6}$ G. Zoth and W. Bergholz, J. Appl. Phys. 67, 6764 (1990).

${ }^{7}$ A. A. Istratov, H. Hieslmair, and E. R. Weber, Appl. Phys. A: Mater. Sci. Process. 70, 489 (2000)

${ }^{8}$ D. Macdonald, T. Roth, P. Deenapanray, K. Bothe, P. Pohl, and J. Schmidt, J. Appl. Phys. 98, 083509 (2005).

${ }^{9}$ H. Reiss, C. S. Fuller, and F. J. Morin, Bell Syst. Tech. J. 35, 535 (1956).

${ }^{10}$ A. Baghdadi, W. M. Bullis, M. C. Croarkin, Y. Li, R. I. Scace, R. W. Series, P. Stallhofer, and M. Watanabe, J. Electrochem. Soc. 136, 2015 (1989).

${ }^{11}$ C. Hässler, H. U. Hofs, W. Koch, G. Stollwerck, A. Müller, D. Karg, and G. Pensl, Mater. Sci. Eng., B 71, 39 (2000).

${ }^{12}$ P. Wagner and J. Hage, Appl. Phys. A: Solids Surf. 49, 123 (1989).

${ }^{13}$ D. Macdonald, L. J. Geerligs, and A. Azzizi, J. Appl. Phys. 95, 1021 (2004).

${ }^{14}$ R. A. Sinton and A. Cuevas, Appl. Phys. Lett. 69, 2510 (1996).

${ }^{15}$ S. Reggiani, M. Valdinoci, L. Colalongo, M. Rudan, G. Baccarani, A. D. Stricker, F. Illien, N. Felber, W. Fichtner, and L. Zullino, IEEE Trans. Electron Devices 49, 490 (2002).

${ }^{16}$ D. B. M. Klaassen, Solid-State Electron. 35, 953 (1992).

${ }^{17}$ D. B. M. Klaassen, Solid-State Electron. 35, 961 (1992).

${ }^{18}$ T. Buonassisi, A. A. Istratov, M. D. Pickett, M. Heuer, J. P. Kalejs, G. Hahn, M. A. Marcus, B. Lai, Z. Cai, S. M. Heald, T. F. Ciszek, R. F. Clark, D. W. Cunningham, A. M. Gabor, R. Jonczyk, S. Narayanan, E. Sauar, and E. R. Weber, Prog. Photovoltaics 14, 513 (2006). 